\title{
Repeated injections of botulinum toxin-A for epiphora in lacrimal drainage disorders: qualitative and quantitative assessment
}

\author{
Chanjoo Ahn ${ }^{1} \cdot$ Sunah Kang ${ }^{1} \cdot$ Ho-Seok Sa $\mathbb{D}^{1}$
}

Received: 2 July 2018 / Revised: 29 November 2018 / Accepted: 30 December 2018 / Published online: 14 February 2019

(c) The Royal College of Ophthalmologists 2019

\begin{abstract}
Purpose To report the outcome of repeated botulinum toxin-A (BTA) injections in the lacrimal glands in patients with epiphora.

Methods We performed retrospective chart review of patients who were injected with 2.5 units of BTA in the lacrimal gland. Epiphora and tear production were assessed by the Munk score and Schirmer-1 test, respectively, pre-injection and at 1 and 3 months post injection. Regarding repeated injections, the effects of the first were compared to those of the second and third injections.

Results Forty-six eyes of 35 patients had an average of 2.3 injections per eye (range, 1-6). The mean Munk score significantly decreased from 3.72 to 1.87 at 1 month $(p<0.001)$ and 2.21 at 3 months $(p<0.001)$ after injection. The mean Schirmer-1 score also significantly decreased from $15.35 \mathrm{~mm}$ to $10.52 \mathrm{~mm}$ at 1 month $(p<0.001)$ and $12.48 \mathrm{~mm}$ at 3 months $(p<0.001)$ after injection. The mean reduction rates of Munk and Schirmer-1 scores after the second $(66.1 \%$ and $29.8 \%$, respectively) and the third injections ( $56.1 \%$ and $23.3 \%$, respectively) were not significantly different from those after the first injection (63.3\% and $26.1 \%$, respectively) ( $p>0.05$ for each comparison). There was a significant correlation between the difficulty in exposing the lacrimal gland for injection and the risk of complication $(p=0.017)$.

Conclusion BTA injection in the lacrimal gland showed favourable outcomes; repeated injections did not compromise efficacy. BTA injection can be safely repeated for epiphora, especially in patients whose lacrimal gland can be easily exposed.
\end{abstract}

\section{Introductions}

Epiphora is a common problem causing blurred vision, eye redness, ocular discomfort, and skin irritation, which can affect quality of life. Obstruction of the tear drainage system is one of the most common causes of epiphora, and surgical procedures such as silicone stent intubation and dacryocystorhinostomy, according to the obstruction site, have been the standard treatments. In particular, conjunctivodacryocystorhinostomy (CDCR) may be required for proximal canalicular obstruction [1], but it has a risk of complications such as tube extrusion, malposition, obstruction, and tearing in the recumbent position $[1,2]$.

Ho-Seok Sa

lineblue@hanmail.net

1 Department of Ophthalmology, University of Ulsan College of Medicine, Asan Medical Center, Seoul, Korea
Several reports described the effects of injection of botulinum toxin-A (BTA) in the lacrimal gland in patients with epiphora [3-8]. BTA injection in the lacrimal gland decreases tear production by blocking presynaptic release of acetylcholine into the neuromuscular end plates of cholinergic nerve fibres [9]. To our knowledge, however, there have been few previous studies that quantitatively assessed the effect of BTA injection or that reported the efficacy of repeated BTA injections. Thus, the purpose of this study was to report the qualitative and quantitative assessment of repeated BTA injections in the lacrimal gland in patients with epiphora.

\section{Materials and methods}

We reviewed the clinical records of all consecutive patients who had BTA injections in the lacrimal gland, performed by one operator (H-S.S.) between March 2011 and July 2017 for epiphora caused by various aetiologies. 
The review included demographic information, previous medical history, aetiology of epiphora, clinical information needed for epiphora assessment, treatment information, outcomes, and complications. Each patient underwent a lacrimal system examination at presentation that included lacrimal irrigation and diagnostic probing, and dacryocystography if needed. Indications for BTA injection included proximal canalicular obstruction, functional nasolacrimal duct obstruction (NLDO), crocodile tearing, and inoperable general condition. All patients included in the study underwent one or more BTA injections in the lacrimal gland and were followed up for at least 3 months. We performed repeated BTA injections when the patient wanted another injection for recurrent epiphora. We excluded patients who were followed up for $<3$ months or who did not have adequate follow-up data.

The severity of epiphora was assessed with the Munk epiphora rating system and the Schirmer-1 test before injection, at 1 month and 3 months after injection, and at the last follow-up visit [10]. The Munk score consisted of 5 grades from 0 to 4 : $0=$ no epiphora; $1=$ occasional epiphora requiring dabbing less than twice a day; $2=$ epiphora requiring dabbing 2-4 times a day; $3=$ epiphora requiring dabbing 5-10 times a day; $4=$ epiphora requiring dabbing $>10$ times a day or constant teaming [10]. In patients with repeated injections, epiphora evaluation was repeated according to the same evaluation methods. The effects of the second and third injections were compared with that of the first injection by comparing the reduction rate of Munk and Schirmer-1 scores after each injection. All patients were asked to report the subjective duration of effect and complications related to BTA injections. The paired $t$ test and Fisher's exact test were conducted for analysing categorical data. Statistical significance was set at $p<0.05$.

BTA (Botox; Allergan; Irvine, CA, USA: 50 mouse unit) was reconstituted with sterile, preservative-free $0.9 \%$ sodium chloride solution diluted to a concentration of 50 units $/ 1 \mathrm{ml}$. Under topical anaesthesia with proparacaine, we gently elevated the lateral portion of the upper eyelid to expose the palpebral lobe of the lacrimal gland while the patient looked down and to the nasal side (easy-exposure group). When the palpebral lobe of patients could not be well exposed with manual elevation of the eyelids, we elevated the upper eyelid with a Desmarres eyelid retractor (difficult-exposure group). A transconjunctival injection of 2.5 units $/ 0.05 \mathrm{ml}$ of BTA using a 30-gauge needle tuberculin syringe was administered into the bulging part of the exposed palpebral lobe.

This retrospective study was approved by the institutional review board at our institution and was carried out following the tenets of the Declaration of Helsinki.
Table 1 Characteristics of 46 eyes of 35 patients who had botulinum toxin-A injections in the lacrimal gland

\begin{tabular}{ll}
\hline Median age (range) (years) & $66(8 \sim 83)$ \\
Male:female & $20: 15$ \\
Unilateral injection:bilateral injection (eyes) & $24: 11$ \\
Median follow-up (range) (months) & $22.0(3 \sim 76)$ \\
Mean number of injections (range) & $2.3(1 \sim 6)$ \\
Mean interval of injections (range) (months) & $6.0(1 \sim 17)$ \\
\hline
\end{tabular}

\section{Results}

Forty-six eyes of 35 patients were included in the study. The median age was 66 years (range, $8-83$ years), and there were 20 males and 15 females. The median follow-up was 22.0 months (range, 3-76 months). A mean of $2.3 \pm 1.5$ injections (median $=2.0$, range, $1-6$ injections) were given per eye. Out of the total 46 eyes, 21 eyes (46\%) underwent only one BTA injection and 25 eyes (54\%) underwent repeated injections: 9 eyes (20\%) had two injections, 4 eyes (9\%) had three injections, and 12 eyes $(26 \%)$ had four or more injections. The mean subjective duration of BTA effect was $3.1 \pm 1.4$ months (range, $1-7$ ) and the mean interval between injections was $6.0 \pm 4.0$ months (range, 1-17) (Table 1).

The most common aetiology of epiphora was proximal canalicular obstruction (32 eyes, 69\%), followed by functional NLDO (6 eyes, 13\%), crocodile tearing ( 4 eyes, $8 \%$ ), and NLDO with inoperable condition (4 eyes, $8 \%$ ). Causes of proximal canalicular obstruction included S-1 anticancer therapy-associated obstruction (15 eyes), idiopathic obstruction ( 6 eyes), traumatic obstruction (6 eyes), postepidemic keratoconjunctivitis obstruction ( 2 eyes), CDCR failure ( 2 eyes), and punctal plug-related obstruction ( 1 eye). The inoperable conditions included 2 eyes with nasal cavity cancer and 2 eyes in insufficient general condition (Table 2). There were patients who had surgical history of silicone stent intubation $(n=3)$, dacryocystorhinostomy $(n=3)$, or CDCR $(n=2)$, but all of the bypass tube including silicone stent and Jones tube had been removed prior to BTA injections. None of the patients had bypass tube at the time of BTA injections and during follow-up.

The mean Munk score significantly decreased from $3.72 \pm 0.7$ (range, 2-4) before injection to $1.87 \pm 0.9$ (range, $0-4 ; p<0.001$, paired $t$ test) at 1 month and $2.21 \pm 1.0$ (range, $0-4 ; p<0.001$, paired $t$ test) at 3 months after BTA injection (Fig. 1a). The mean Schirmer-1 score also significantly decreased from $15.35 \pm 4.3 \mathrm{~mm}$ (range, 6-24) before injection to $10.52 \pm 3.2 \mathrm{~mm}$ (range, $5-22 ; p<0.001$, paired $t$ test) at 1 month and $12.48 \pm 3.8 \mathrm{~mm}$ (range, 6-20; $p<0.001$, paired $t$ test) at 3 months after injection (Fig. 1b).

In terms of efficacy and duration of effect of repeated injections, there was no significant difference in the mean reduction rates of the Munk and Schirmer-1 scores and the 
Table 2 Aetiologies of epiphora in 46 eyes

\begin{tabular}{lc}
\hline Aetiology & Eyes, $n(\%)$ \\
\hline Proximal canalicular obstruction & $32(69.6)$ \\
Oral S-1-associated & $15(32.6)$ \\
Idiopathic & $6(13.0)$ \\
Traumatic & $6(13.0)$ \\
Post-EKC & $2(4.3)$ \\
CDCR failure & $2(4.3)$ \\
Punctal plug-related & $1(2.2)$ \\
Functional NLDO & $6(13.0)$ \\
Crocodile tearing & $4(8.7)$ \\
Inoperable condition & $4(8.7)$ \\
Nasal cavity cancer & $2(4.3)$ \\
Insufficient general condition & $2(4.3)$ \\
Total & $46(100)$ \\
\hline
\end{tabular}

$E K C$ epidemic keratoconjunctivitis; $C D C R$ conjunctivodacryocystorhinostomy; $N L D O$ nasolacrimal duct obstruction

mean subjective duration of effect after each injection (the first, $n=46$ eyes; the second, $n=25$ eyes; the third, $n=16$ eyes). The mean reduction rate of the Munk score after the first injection was $63.3 \pm 16.0 \%$ (range, 50-100\%), which was not significantly different from the mean reduction rate after the second injection $(66.1 \pm 26.1 \%$; range, $25-100 \%$; $p=0.657$, paired $t$ test) and after the third injection (56.1 \pm 16.6\%; range, 50-75\%; $p=0.085$, paired $t$ test) (Fig. 2a). Similarly, the mean reduction rate of the Schirmer-1 score after the first injection was $26.1 \pm 14.9 \%$ (range, $-8.3-54.2 \%$ ), which was not significantly different from the reduction rate after the second injection $(29.8 \pm 20.5 \%$; range, $0-62.5 \% ; p=0.416$, paired $t$ test) and after the third injection $(23.3 \pm 21.6 \%$; range, $-23.1-43.8 \% ; p=0.370$, paired $t$ test $)$ (Fig. 2b). The mean subjective duration of effect after the first injection was $3.2 \pm 1.5$ months (range, 1-6), which was not significantly different from subjective duration of effect after the second injection $(2.8 \pm 1.1$ months; range $1-5 ; p=0.959$, paired $t$ test) and after the third injection $(3.1 \pm 1.6$ months; range, $1-7 ; p=0.437$, paired $t$ test).

Complications occurred in 10 out of the total 106 injections (9.4\%), involving 10 patients, each of whom experienced one complication: seven cases of upper eyelid ptosis and three cases of horizontal diplopia. Of the seven cases of ptosis, two were ptosis obscuring the visual axis and the other five were mild ptosis that did not obscure the visual axis. Ptosis resolved spontaneously within 3 weeks in all seven cases. Diplopia also resolved spontaneously within 6 weeks in all three cases: two in 2 weeks, and the other in 6 weeks. Regarding difficulty in exposing the lacrimal gland at the time of injections, there were 41 eyes (101 injections) in the easy-exposure group and 5 eyes (5 injections) in the difficult-exposure group, which required a Desmarres eyelid retractor owing to difficulty in exposing the lacrimal gland with manual traction of the upper

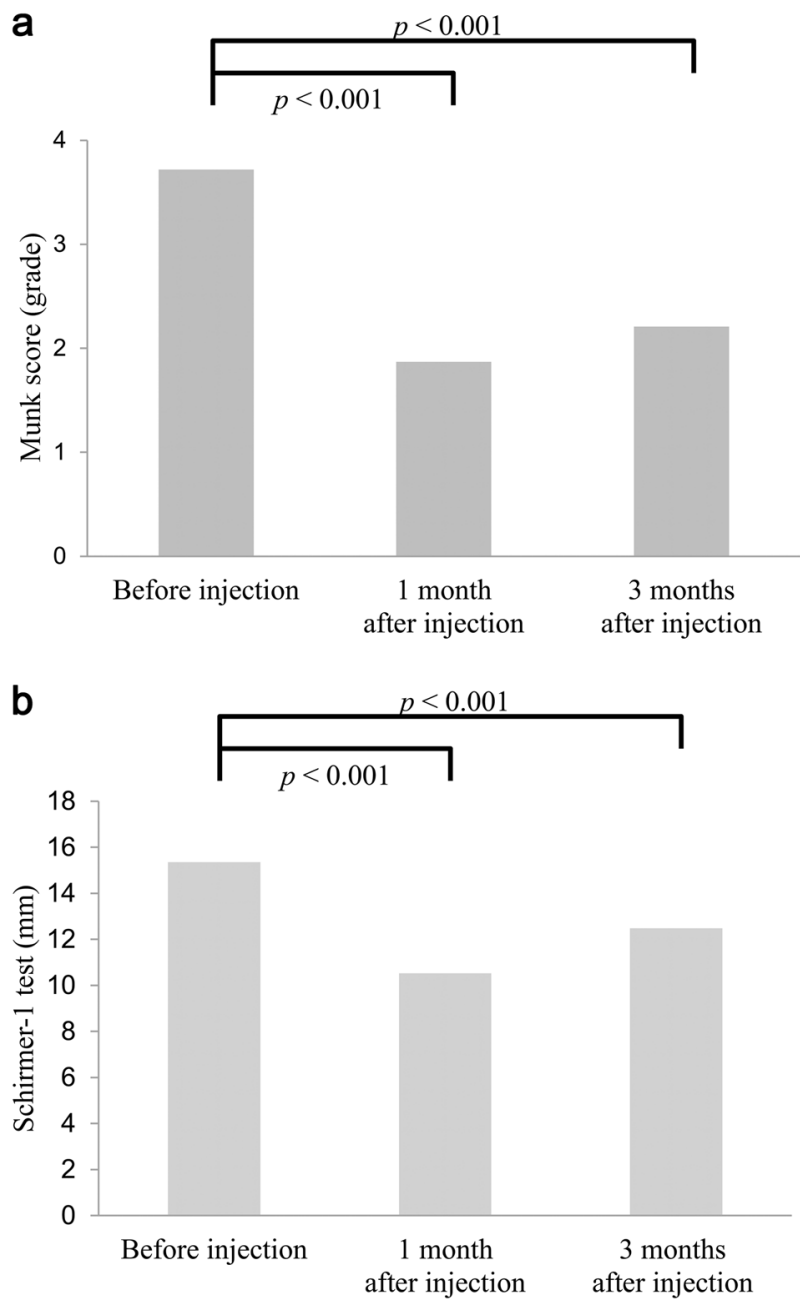

Fig. 1 Change in the mean Munk and Schirmer-1 scores after BTA injection. a The mean Munk score significantly decreased at 1 month $(1.87 \pm 0.9, p<0.001)$ and 3 months $(2.21 \pm 1.0, p<0.001)$ after BTA injection compared with before injection $(3.72 \pm 0.7)$. b The mean Schirmer-1 score significantly decreased at 1 month $(10.52 \pm 3.2, p<$ $0.001)$ and 3 months $(12.48 \pm 3.8, p<0.001)$ after BTA injection compared with the score before injection $(15.35 \pm 4.3)$

eyelid alone. Complications including ptosis and diplopia occurred in 6 out of 101 injections in the easy-exposure group, and in 4 out of 5 injections in the difficult-exposure group. There was a significant association between the difficulty in exposing the lacrimal gland for injection and the risk of complication ( $p=0.017$, Fisher's exact test).

\section{Discussion}

The major finding of this study, using both Munk and Schirmer score methods, is that BTA injections in the lacrimal gland can improve epiphora both qualitatively and quantitatively, without permanent complications. We also found the effect of injection and the duration of effect remained significantly consistent in repeated injections. 

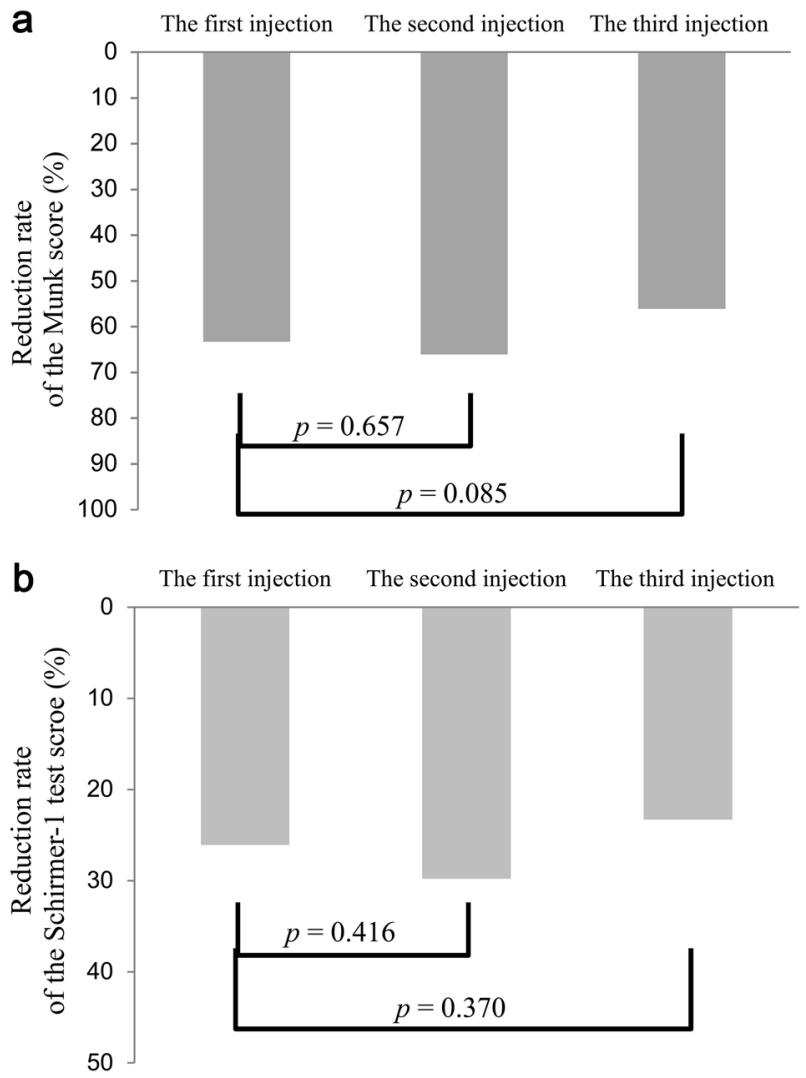

Fig. 2 Comparison of the mean reduction rates of Munk and Schirmer1 scores between before and after each injection in patients who had repeated injections. a The mean reduction rate of Munk score after the first injection $(63.3 \pm 16.0 \%)$ was not significantly different from the mean reduction rate after the second injection $(66.1 \pm 26.1 \% ; p=$ $0.657)$ and after the third injection $(56.1 \pm 16.6 \% ; p=0.085)$. b The mean reduction rate of Schirmer-1 score after the first injection (26.1 \pm $14.9 \%$ ) was not significantly different from the mean reduction rate after the second injection $(29.8 \pm 20.5 \% ; p=0.416)$ and after the third injection $(23.3 \pm 21.6 \% ; \mathrm{p}=0.370)$

Both qualitative and quantitative evaluations are important in patients with epiphora, but there have been few studies reporting the effect of BTA injection with both assessment methods. Ziahosseini et al. [7] evaluated the effect of BTA injection for epiphora by the reduction of the mean Munk score after injection from 3.4 to 1.6 in the series with 22 eyes. Kaynak et al. [8] reported both qualitative and quantitative evaluations of epiphora in a comparative study including 20 eyes treated with BTA injection: the mean Munk and Schirmer-1 scores were 3.95 and $12 \mathrm{~mm}$ before injection respectively, and decreased to 1.05 and $5.4 \mathrm{~mm}$ at 1 month after injection and to $1.36 \mathrm{~mm}$ and $5.8 \mathrm{~mm}$ at 3 months after injection, respectively. These results of previous studies were similar to our findings showing significant improvement of epiphora at 1 month and 3 months after BTA injection [3-8].

To our knowledge, there have been few studies on the clinical outcomes of repeated BTA injections in the lacrimal gland, and our study revealed that each injection shows a consistent efficacy and duration of effect on improving epiphora when BTA is injected repeatedly in the lacrimal gland. Owing to the pharmacological characteristics of BTA, the treated muscles or secretary glands gradually regain function and generally return to their original status approximately 3 months after injection, and repeated injections may be required when the patient's symptom recurs and additional treatment is needed [11-14]. Some previous studies evaluated the histologic changes of lacrimal gland after BTA injection in the lacrimal gland of rabbits, and showed that BTA injections were not associated with inflammation or structural changes of acini or ductules such as atrophy and fibrosis [9, 15]. BTA is also widely used for treating limb spasticity, and previous studies demonstrated a consistent efficacy and safety of repeated BTA injections in patients with upper and lower limb spasticity $[16,17]$. Taken together, these findings suggest that BTA can be injected in the lacrimal gland repeatedly at the same dosage if needed, and each injection may have a similar efficacy for treating epiphora.

The most common complications of BTA injection in the lacrimal gland are ptosis and diplopia; these are typically temporary, lasting for 2-4 weeks [18]. Diffusion of toxin into the levator palpebrae superioris muscle and superior or lateral rectus muscles is believed to cause upper eyelid ptosis and diplopia, respectively $[3,5,7,8]$. The complication rate was $9.4 \%$ (10/106 injections) in our study, and the reported rates vary among studies, from $11 \%$ to $25 \%$ $[3,5,7,8]$. Interestingly, we found a significant correlation between the difficulty in exposing the lacrimal gland and the risk of complication $(p=0.017)$. Given the complications caused by toxin diffusion, if it is difficult to expose the lacrimal gland, the BTA cannot be injected correctly into the lacrimal gland and is likely to spread to surrounding tissues such as levator and rectus muscles. Our patients who had difficulty in exposing the lacrimal gland had only one BTA injection each, and it was not confirmed whether these patients would experience complications repeatedly if they got repeated injections. Nevertheless, our findings suggest that physicians should inject BTA into the most protruding part of the lacrimal gland after the maximum exposure of the gland to avoid unwanted toxin spread and to reduce the incidence of complications. We believe that transconjunctival BTA injection method is particulary useful in older patients who may have horizontally loose upper eyelid and whose lacrimal gland can be easily exposed.

This study had several limitations. First, this study was based on data retrospectively reviewed. There is the potential for error in collecting data on the duration of effect or complications assessed by patients' reports. However, all patients included in the study were managed and evaluated using a uniform and standardised method by one practitioner, and patients with missing data or short follow-up 
periods were excluded. Second, approximately half of the patients in this study underwent only one BTA injection. However, this series involving 46 eyes represents one of the largest single-centre studies of BTA injection in the lacrimal gland with a long follow-up period (median of 22 months), and we were able to perform a comparative analysis to evaluate the efficacy of repeated injections: this is a main strength of our results in this study.

In conclusion, transconjunctival injection of BTA in the lacrimal gland showed favourable outcomes for epiphora without permanent complications. It is a simple and minimally invasive technique performed in the office. Repeated injections had consistent efficacy and duration of effect. BTA injections can be repeated for symptomatic epiphora as an alternative to complex surgery such as CDCR, especially in older patients whose lacrimal gland can be easily exposed.

\section{Summary}

\section{What was known before}

- Obstruction of the tear drainage system is one of the most common causes of epiphora, and surgical procedures have been the standard treatments.

- Several reports described the effects of injection of BTA in the lacrimal gland in patients with epiphora.

- However, there have been few previous studies that quantitatively assessed the effect of BTA injection or that reported the efficacy of repeated BTA injections.

\section{What this study adds}

- Transconjunctival injection of BTA in the lacrimal gland showed favourable outcomes for epiphora without permanent complications.

- Repeated injections had consistent efficacy and duration of effect, and BTA injections can be repeated for symptomatic epiphora as an alternative to complex surgery.

- There was a correlation between the difficulty in exposing the lacrimal gland with manual elevation of upper eyelids and the risk of complications.

Acknowledgements The authors have no proprietary interests in or financial support for the development or marketing of instruments or equipment mentioned in this article or any competing instruments or equipment.

\section{Compliance with ethical standards}

Conflict of interest The authors declare that they have no conflict of interest.
Publisher's note: Springer Nature remains neutral with regard to jurisdictional claims in published maps and institutional affiliations.

\section{References}

1. Lim C, Martin P, Benger R, Kourt G, Ghabrial R. Lacrimal canalicular bypass surgery with the Lester Jones tube. Am J Ophthalmol. 2004;137:101-8.

2. Athanasiov PA, Madge S, Kakizaki H, Selva D. A review of bypass tubes for proximal lacrimal drainage obstruction. Surv Ophthalmol. 2011;56:252-66.

3. Whittaker KW, Matthews BN, Fitt AW, Sandramouli S. The use of botulinum toxin $\mathrm{A}$ in the treatment of functional epiphora. Orbit. 2003;22:193-8.

4. Tu AH, Chang EL. Botulinum toxin for palliative treatment of epiphora in a patient with canalicular obstruction. Ophthalmology. 2005;112:1469-71.

5. Wojno TH. Results of lacrimal gland botulinum toxin injection for epiphora in lacrimal obstruction and gustatory tearing. Ophthal Plast Reconstr Surg. 2011;27:119-21.

6. Eustis HS, Babiuch A. Botulinum toxin injection into the lacrimal gland for treatment of proximal nasolacrimal duct obstructions in children. J Pediatr Ophthalmol Strabismus. 2014;51:e75-77. Online

7. Ziahosseini K, Al-Abbadi Z, Malhotra R. Botulinum toxin injection for the treatment of epiphora in lacrimal outflow obstruction. Eye (Lond). 2015;29:656-61.

8. Kaynak P, Karabulut GO, Ozturker C, Fazil K, Arat YO, Perente $\mathrm{I}$, et al. Comparison of botulinum toxin-A injection in lacrimal gland and conjunctivodacryocystorhinostomy for treatment of epiphora due to proximal lacrimal system obstruction. Eye (Lond). 2016;30:1056-62.

9. Demetriades AM, Leyngold IM, D'Anna S, Eghrari AO, Emmert DG, Grant MP, et al. Intraglandular injection of botulinum toxin a reduces tear production in rabbits. Ophthal Plast Reconstr Surg. 2013;29:21-24.

10. Munk PL, Lin DT, Morris DC. Epiphora: treatment by means of dacryocystoplasty with balloon dilation of the nasolacrimal drainage apparatus. Radiology. 1990;177:687-90.

11. Holds JB, Alderson K, Fogg SG, Anderson RL. Motor nerve sprouting in human orbicularis muscle after botulinum A injection. Invest Ophthalmol Vis Sci. 1990;31:964-7.

12. Bambrick L, Gordon T. Acetylcholine receptors and sodium channels in denervated and botulinum-toxin-treated adult rat muscle. J Physiol. 1987;382:69-86.

13. Thesleff S, Molgo J, Tagerud S. Trophic interrelations at the neuromuscular junction as revealed by the use of botulinal neurotoxins. J Physiol (Paris). 1990;84:167-73.

14. Dutton JJ, Fowler AM. Botulinum toxin in ophthalmology. Surv Ophthalmol. 2007;52:13-31.

15. Kim JW, Baek S. Functional and histologic changes in the lacrimal gland after botulinum toxin injection. J Craniofac Surg. 2013;24:1960-9.

16. Santamato A, Panza F, Intiso D, Baricich A, Picelli A, Smania N, et al. Long-term safety of repeated high doses of incobotulinumtoxinA injections for the treatment of upper and lower limb spasticity after stroke. J Neurol Sci. 2017;378:182-6.

17. Gracies JM, O'Dell M, Vecchio M, Hedera P, Kocer S, Rudzinska-Bar M, et al. Effects of repeated abobotulinumtoxinA injections in upper limb spasticity. Muscle Nerve. 2018; 57:245-54.

18. Singh S, Ali MJ, Paulsen F. A review on use of botulinum toxin for intractable lacrimal drainage disorders. Int Ophthalmol. 2018;38:2233-2238. 